\title{
ON PIECEWISE-LINEAR HOMEOMORPHISMS BETWEEN DISTRIBUTIVE AND ANTI-BLOCKING POLYHEDRA
}

\author{
CHRISTOPH PEGEL AND RAMAN SANYAL
}

\begin{abstract}
Stanley (1986) introduced the order polytope and chain polytope of a partially ordered set and showed that they are related by a piecewise-linear homeomorphism. In this paper we view order and chain polytopes as instances of distributive and anti-blocking polytopes, respectively. Both these classes of polytopes are defined in terms of the componentwise partial order on $\mathbb{R}^{n}$. We generalize Stanley's PL-homeomorphism to a large class of distributive polyhedra using infinite walks in marked networks.
\end{abstract}

\section{INTRODUCTION}

Let $(P, \preceq)$ be a finite partially ordered set (poset, for short). Stanley [22] introduced two convex polytopes associated to $P$, the order polytope

$$
\mathcal{O}(P):=\left\{f \in \mathbb{R}^{P}: \begin{array}{cc}
0 \leq f(a) \leq 1 & \text { for all } a \in P \\
f(a) \leq f(b) & \text { for all } a \prec b
\end{array}\right\}
$$

and the chain polytope

$$
\mathcal{C}(P):=\left\{g \in \mathbb{R}^{P}: \begin{array}{cl}
g(a) \geq 0 & \text { for all } a \in P \\
g\left(a_{1}\right)+g\left(a_{2}\right)+\cdots+g\left(a_{k}\right) \leq 1 & \text { for all } a_{1} \prec a_{2} \prec \cdots \prec a_{k}
\end{array}\right\} .
$$

The poset can be completely recovered from $\mathcal{O}(P)$ and many geometric properties of $\mathcal{O}(P)$ can be translated into combinatorial properties of $P$. In particular, the Ehrhart polynomial of $\mathcal{O}(P)$ is the order polynomial of $P$ and the normalized volume $(|P|) ! \cdot \operatorname{vol}(\mathcal{O}(P))$ is the number of linear extensions of $P$. We refer the reader to Stanley's original paper and [3, Ch. 6] for more details. So it is fair to say that the order polytope $\mathcal{O}(P)$ gives a geometric representation of $P$. The chain polytope, on the other hand, is defined in terms of the comparability graph $G_{P}=(P, E)$ of $P$. Two elements $a, b \in P$ share an edge in $G$ if and only if $a \prec b$ or $b \prec a$. Chains in $P$ correspond to cliques in $G$. The comparability graph can be recovered from $\mathcal{C}(P)$ but $P$ is in general not determined by $G_{P}$. Stanley defines a piecewise-linear (PL) homeomorphism $\phi: \mathbb{R}^{P} \rightarrow \mathbb{R}^{P}$ called the transfer map that is volume- and lattice preserving and that maps $\mathcal{O}(P)$ to $\mathcal{C}(P)$. This shows, quite unexpectedly, that both polytopes have the same Ehrhart polynomial and normalized volume and, consequently, that order polynomial and number of linear extensions only depend on the comparability graph. Order and chain polytopes have many applications in combinatorics as well as in geometry and, together with their connecting PL-homeomorphism, have been generalized to marked posets [1, 15, 21], to marked chain-order polytopes [9, 10], and to double posets [8], to name a few. The aim of this paper is to give a generalization of Stanley's transfer map to a larger class of geometric objects that we now define.

Date: August 5, 2021.

2010 Mathematics Subject Classification. 52B12, 05C20, 52A41.

Key words and phrases. order polytopes, chain polytopes, distributive polyhedra, anti-blocking polyhedra, piecewise-linear maps, marked networks. 
Let $V$ be some finite set and $\mathbb{R}^{V}$ equipped with the usual componentwise partial order $\leq$. A convex polyhedron $Q \subseteq \mathbb{R}_{\geq 0}^{V}$ is called anti-blocking [14] or a convex corner [4] if for $y \in Q$ and $x \in \mathbb{R}_{\geq 0}^{V}$

$$
x \leq y \quad \Longrightarrow \quad x \in Q .
$$

The chain polytope is easily seen to be anti-blocking. An order ideal in a poset is a subset that is down-closed with respect to the partial order. Condition (3) thus states that anti-blocking polyhedra can be viewed as convex order ideals in $\left(\mathbb{R}_{\geq 0}^{n}, \leq\right)$.

For $x, y \in \mathbb{R}^{V}$, let us write $x \wedge y$ and $x \vee y$ for the coordinate-wise minimum and maximum, respectively. In particular, $\left(\mathbb{R}^{V}, \wedge, \vee\right)$ is an (infinite) distributive lattice with meet $\wedge$ and join $\vee$. It is straightforward to verify that $\mathcal{O}(P)$ is closed under meets and joins. Thus $\mathcal{O}(P)$ is a polyhedron as well as a sublattice of $\mathbb{R}^{P}$. Such polyhedra were introduced by Felsner and Knauer [12] under the name distributive polyhedra. Felsner and Knauer noted that order polytopes and, more generally, alcoved polytopes [19] are distributive. Since marked order polytopes are coordinate sections of dilated order polytopes, they are automatically distributive. There are many other polyhedra in combinatorics that turn out to be distributive. For example, the $t$-Cayley and $t$-Gayley polytopes of Konvalinka and Pak [17], the s-lecture hall polytopes and cones of Bousquet-Mélou and Eriksson [5, 6], and their poset generalizations due to Brändén-Leander [7]. See Section 5 for more on these classes of examples.

Stanley's piecewise-linear homeomorphism connects the distributive polytopes $\mathcal{O}(P)$ to the antiblocking polytope $\mathcal{C}(P)$ with phenomenal combinatorial consequences. Similar PL-maps have been constructed in other contexts. For example, the polytope $P_{n}(x)$ studied by Pitman-Stanley is an anti-blocking polytope and a linear isomorphism to a distributive polytope is constructed in [24, Sect. 4]. Beck, Braun, and Le [2] introduced Cayley polytopes $C_{n}$ (denoted by $\mathbf{A}_{n}$ in [18]) as

$$
C_{n}=\left\{x \in \mathbb{R}^{n}: 1 \leq x_{i} \leq 2 x_{i-1} \text { for all } 1 \leq i \leq n\right\},
$$

where $x_{0}:=1$. This is a distributive polytope. In [18], Konvalinka and Pak define an anti-blocking polytope $\mathbf{Y}_{n}$ as the set of all $y \in \mathbb{R}^{n}$ with $y \geq 0$ and for all $1 \leq h \leq n$

$$
\sum_{j=1}^{h} 2^{h-j} y_{j} \leq 2^{h}-1
$$

and a linear lattice-preserving map $\phi: \mathbb{R}^{n} \rightarrow \mathbb{R}^{n}$ with $\phi\left(\mathbf{Y}_{n}\right)=C_{n}$ to give a simple proof of a conjecture of Braun on partitions [2].

In this paper, we study the relation between distributive and anti-blocking polyhedra more closely and we construct PL-homeomorphisms for a large class of distributive polyhedra that subsumes marked order polyhedra. Our PL-maps generalize Stanley's original construction as well as the mentioned examples and depends on the convergence of series given by infinite walks in directed networks. Most of the work presented here also appeared in the first authors $\mathrm{PhD}$ thesis [20].

Acknowledgements. The second author wants to thank Kolja Knauer and Martin Skutella for fruitful discussions.

\section{Distributive POLYHEDRA AND MARKED NETWORKS}

A marked network is a tuple $\Gamma=(V \uplus A, E, \alpha, c, \lambda)$. It consists of a finite loop-free directed multigraph $(V \uplus A, E)$ on nodes $V \uplus A$ with edges $E$. We refer to the nodes in $A$ as marked nodes with marking $\lambda \in \mathbb{R}^{A}$. To every directed edge $v \stackrel{e_{\rightarrow}}{w} w$ there are two associated weights $\alpha_{e}, c_{e} \in \mathbb{R}$ with $\alpha_{e}>0$. In drawings of a marked network, we will depict an edge $v \stackrel{e}{\rightarrow} w$ with weights $\alpha_{e}$ and $c_{e}$ as

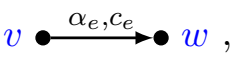


where blue labels are node names. Marked nodes are drawn as squares with red labels and when edge weights are omitted, we always assume $\alpha_{e}=1$ and $c_{e}=0$. See Figures 1 and 2a.

To a marked network, we associate the polyhedron $\mathcal{O}(\Gamma) \subseteq \mathbb{R}^{V}$ consisting of all points $x \in \mathbb{R}^{V}$ such that

$$
\alpha_{e} x_{w}+c_{e} \leq x_{v} \text { for all edges } v \stackrel{e}{\rightarrow} w,
$$

where we set $x_{v}:=\lambda_{v}$ for $v \in A$.

Example 2.1 (Marked order polyhedra). For a poset $(P, \preceq)$ let $\widehat{P}=P \uplus\{\hat{0}, \hat{1}\}$ be the poset with minimum $\hat{0}$ and maximum $\hat{1}$. A marked network is obtained from the Hasse diagram of $\widehat{P}$ with $A:=\{\hat{0}, \hat{1}\}, V:=P$, and $E$ consisting of edges $v \rightarrow w$ for $w$ covered by $v$. Setting $\alpha \equiv 1, c \equiv 0$ and $\left(\lambda_{\hat{0}}, \lambda_{\hat{1}}\right)=(0,1)$, we obtain the order polytope $\mathcal{O}(P)$. By allowing more general $A$, this yields the marked order polyhedra $[1,21]$.

In a similar fashion one sees that the Cayley polytope $C_{n}$ is also of the form $\mathcal{O}(\Gamma)$ for the simple network given in Figure 1. It is straightforward to verify that $\mathcal{O}(\Gamma)$ is a distributive polyhedron. The main result in [12] is a characterization of distributive polyhedra in terms of marked networks.

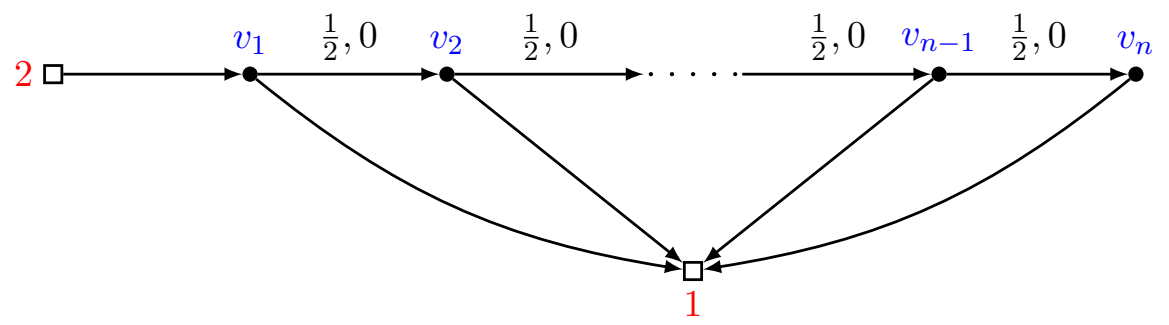

FigurE 1. The marked network defining the Cayley polytope $C_{n}$.

Theorem 2.2 ([12, Thm. 4]). Every distributive polyhedron is of the form $\mathcal{O}(\Gamma)$ for some marked network $\Gamma$.

Remark 2.3. In order to make the structural similarity to (marked) order polyhedra more explicit, our definition of marked network is slightly different from that employed in [12]. Most notably, we use markings and require $\alpha_{e}>0$ instead of allowing loops and our edge weights $\left(\alpha_{e}, c_{a}\right)$ translate to $\left(\frac{1}{\alpha_{e}}, \frac{-c_{e}}{\alpha_{e}}\right)$ in the notation of [12].

To a marked network with at least all sinks marked, we associate the transfer map $\phi_{\Gamma}: \mathbb{R}^{V} \rightarrow \mathbb{R}^{V}$ defined as

$$
\phi_{\Gamma}(x)_{v}:=x_{v}-\max _{v \stackrel{e}{\rightarrow} w}\left(\alpha_{e} x_{w}+c_{e}\right) .
$$

Let us point out again, that $x_{v}=\lambda_{v}$ for $v \in A$.

If $\Gamma$ is derived from a poset $P$ as in Example 2.1, the map $\phi_{\Gamma}$ is the original transfer map from [22]. If $\Gamma$ is acyclic, that is, the underlying directed graph has no directed cycles, then we will see in Theorem 3.5 that $\phi_{\Gamma}$ is bijective. In the non-acyclic situation, this need not be true. In order to illustrate, let us give a geometric reformulation of the transfer map. We denote the standard basis of $\mathbb{R}^{V}$ by $\left\{e_{v}\right\}_{v \in V}$. For a polyhedron $Q \subseteq \mathbb{R}^{V}$ that does not contain $-e_{v}$ in its recession cone for all $v \in V$, define the map $\phi_{Q}: Q \rightarrow \mathbb{R}^{V}$ by

$$
\phi_{Q}(x)_{v}:=\max \left(\mu \geq 0: x-\mu e_{v} \in Q\right)
$$

for all $v \in V$. If $Q$ is defined by linear inequalities of the form $\ell_{i}(x) \leq b_{i}$, then

$$
\phi_{Q}(x)_{v}=\min \left(\frac{b_{i}-\ell_{i}(x)}{-\ell_{i}\left(e_{v}\right)} \text { : for } i \text { with } \ell_{i}\left(e_{v}\right)<0\right) \text {. }
$$




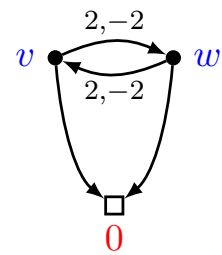

(a) Network $\Gamma$

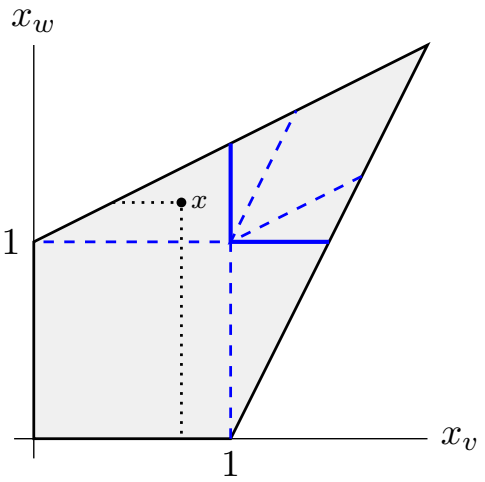

(b) the polytope $\mathcal{O}(\Gamma)$

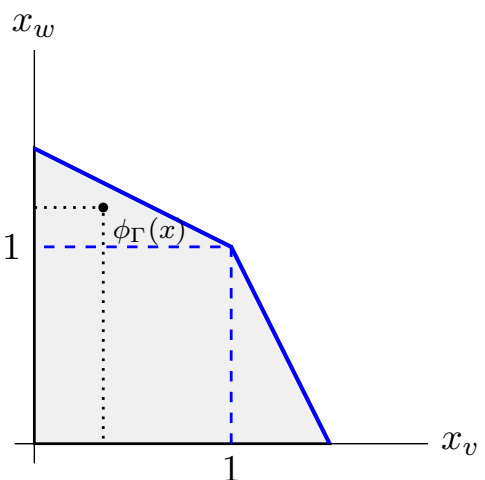

(c) the image $\phi_{\Gamma}(\mathcal{O}(\Gamma))$

Figure 2. The marked network $\Gamma$ of Example 2.4 with the associated distributive polytope and its "folded" image under the non-injective transfer map.

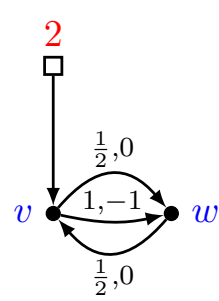

(a) a network $\Gamma$

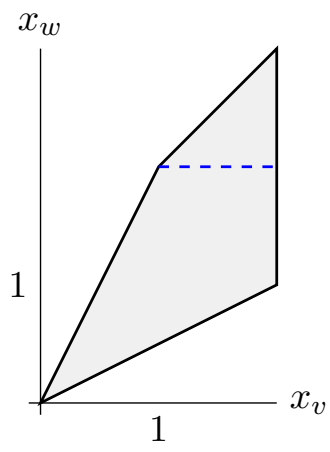

(b) the polytope $\mathcal{O}(\Gamma)$

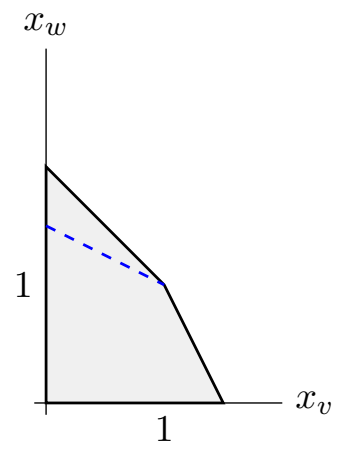

(c) the image $\phi_{\Gamma}(\mathcal{O}(\Gamma))$

Figure 3. The marked network $\Gamma$ of Example 2.5 with the associated distributive polytope and its bijective image under the transfer map.

From (4), we conclude that $\phi_{\mathcal{O}(\Gamma)}$ coincides with $\phi_{\Gamma}$.

Example 2.4. Let $\Gamma$ be the marked network depicted in Figure 2a. The distributive polyhedron $\mathcal{O}(\Gamma)$ is a "kite" given by the inequalities $0 \leq x_{v}, 0 \leq x_{w}, 2 x_{v}-2 \leq x_{w}$ and $2 x_{w}-2 \leq x_{v}$ as shown in Figure $2 b$. The transfer map for this network is given by

$$
\phi_{\Gamma}\left(\begin{array}{l}
x_{v} \\
x_{w}
\end{array}\right)=\left(\begin{array}{l}
x_{v}-\max \left\{0,2 x_{w}-2\right\} \\
x_{w}-\max \left\{0,2 x_{v}-2\right\}
\end{array}\right) .
$$

The transfer map is not injective on $\mathcal{O}(\Gamma)$. For example the vertices $(0,0)$ and $(2,2)$ both get mapped to the origin. In fact, the map is 2-to- 1 and "folds" the polytope along the thick blue line in Figure $2 \mathrm{~b}$. The dashed lines in the lower left part stay fixed under the transfer map and have the same image as the dashed lines in the upper right part. The geometric behavior of the transfer map given above is shown for some $x \in \mathcal{O}(\Gamma)$ using dotted lines.

Example 2.5. Let $\Gamma$ be the marked network depicted in Figure 3a. The distributive polyhedron $\mathcal{O}(\Gamma)$ is a quadrilateral given by the inequalities $\frac{1}{2} x_{v} \leq x_{w}, \frac{1}{2} x_{w} \leq x_{v}, x_{w}-1 \leq x_{v}$ and $x_{v} \leq 2$ as shown in Figure 3b. The transfer map for this network is given by

$$
\phi_{\Gamma}\left(\begin{array}{c}
x_{v} \\
x_{w}
\end{array}\right)=\left(\begin{array}{c}
x_{v}-\max \left\{\frac{1}{2} x_{w}, x_{w}-1\right\} \\
x_{w}-\frac{1}{2} x_{v}
\end{array}\right) .
$$


In this example, the transfer map is bijective and maps $\mathcal{O}(\Gamma)$ to the anti-blocking polytope depicted in Figure 3c. The dashed line divides $\mathcal{O}(\Gamma)$ into the two linearity regions of the transfer map. We will come back to this example in Section 4 after constructing inverse transfer maps and describing the inequalities for $\phi_{\Gamma}(\mathcal{O}(\Gamma))$.

As we have seen in Examples 2.4 and 2.5, some cyclic networks lead to bijective transfer maps while others do not. The important difference in the two examples is the product of weights along the cycles. This motivates the following definition.

Definition 2.6. Let $\Gamma=(V \uplus A, E, \alpha, c, \lambda)$ be a marked network. A walk $W$ in $\Gamma$ is a sequence

$$
W=v_{1} \stackrel{e_{1}}{\rightarrow} v_{2} \stackrel{e_{2}}{\rightarrow} \cdots \stackrel{e_{r}}{\longrightarrow} v_{r+1} \cdots
$$

If $W$ is finite, then its length $|W|$ is the number of edges. The weight of $W$ is

$$
\alpha(W):=\prod_{i=1}^{r} \alpha_{e_{i}} .
$$

If all nodes are distinct, then $W$ is called a path. If all nodes are distinct except for $v_{r+1}=v_{1}$, then we call $W$ a cycle. In accordance with [12], a cycle $C$ is called gainy if $\alpha(C)<1$, lossy if $\alpha(C)>1$ and breakeven if $\alpha(C)=1$. Finally, we call a marked network gainy/lossy/breakeven if all cycles are gainy/lossy/breakeven.

In the following section, we will show that the observation made in Examples 2.4 and 2.5 is true in general: when $\Gamma$ contains only gainy cycles, the transfer map is bijective.

\section{Gainy NETWORKS AND Infinite WALKS}

Throughout this section we assume that $\Gamma=(V \uplus A, E, \alpha, c, \lambda)$ is a gainy marked network such that every sink is marked. Our goal is to construct an inverse to the transfer map $\phi_{\Gamma}$ and show that the image $\phi_{\Gamma}(\mathcal{O}(\Gamma))$ is an anti-blocking polyhedron by giving explicit inequalities determined by walks in $\Gamma$.

Definition 3.1. To $\Gamma$ associate the set $\mathcal{W}$ consisting of finite walks

$$
v_{1} \stackrel{e_{1}}{\rightarrow} v_{2} \stackrel{e_{2}}{\rightarrow} \cdots \stackrel{e_{r}}{\rightarrow} v_{r+1} \quad \text { with } v_{i} \in V \text { for } i \leq r \text { and } v_{r+1} \in A,
$$

as well as infinite walks

$$
v_{1} \stackrel{e_{1}}{\rightarrow} v_{2} \stackrel{e_{2}}{\rightarrow} v_{3} \stackrel{e_{3}}{\rightarrow} \cdots \quad \text { with all } v_{i} \in V .
$$

In particular, $A \subseteq \mathcal{W}$, since walks of length 0 are allowed.

Given a walk $W \in \mathcal{W}$ starting in $w$ and an edge $v \stackrel{e}{\rightarrow} w$ from an unmarked node $v \in V$, denote by $v \stackrel{e}{\rightarrow} W$ the walk in $\mathcal{W}$ obtained by prepending the edge $e$.

In order to define the inverse transfer map, we want to associate to each walk $W \in \mathcal{W}$ an affine-linear form $\Sigma(W)$ on $\mathbb{R}^{V}$ satisfying $\Sigma(a)(x):=\lambda_{a}$ for all trivial walks at a marked element $a \in A$ and for all walks $W=v \rightarrow W^{\prime}$ of positive length, the recursion

$$
\Sigma(W)(x)=\alpha_{e} \Sigma\left(W^{\prime}\right)(x)+\left(x_{v}+c_{e}\right) .
$$

In order to see that $\Sigma$ is well-defined on infinite walks, we need the following statement on convergence of infinite series. 

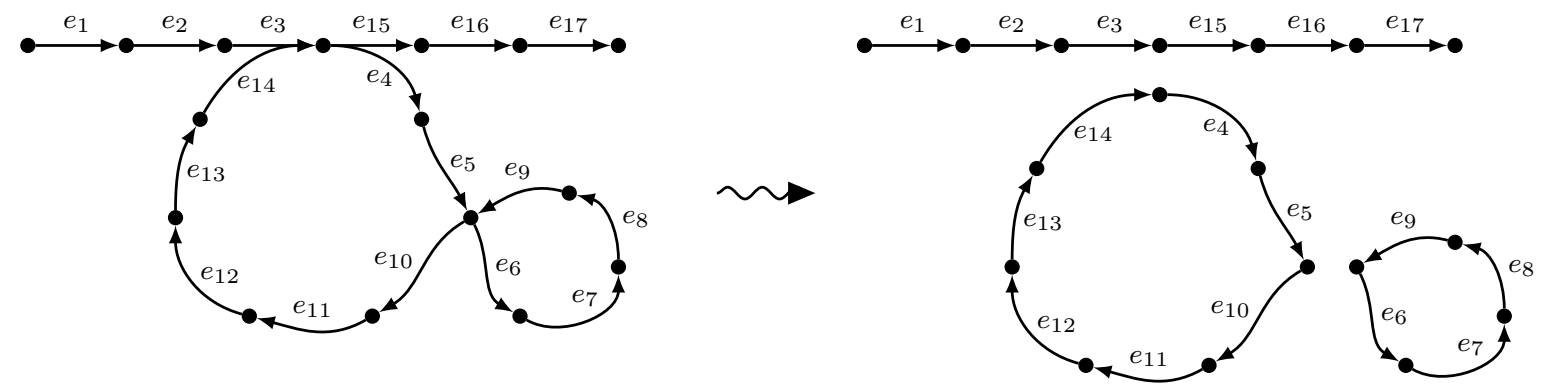

Figure 4. The decomposition of a finite walk into a path and cycles as used in the proof of Proposition 3.2.

Proposition 3.2. Let $W \in \mathcal{W}$ be an infinite walk as in (8). The infinite series

$$
\sum_{k=1}^{\infty}\left(\prod_{j=1}^{k-1} \alpha_{e_{j}}\right)\left(x_{v_{k}}+c_{e_{k}}\right)
$$

absolutely converges for all $x \in \mathbb{R}^{V}$.

Proof. Since $\Gamma$ has only finitely many nodes and edges, we have $\left|x_{v_{k}}+c_{e_{k}}\right| \leq M$ for some $M$. It is therefore enough to show absolute convergence of $\sum_{k=1}^{\infty} \prod_{j=1}^{k-1} \alpha_{e_{j}}$. Using the root test, it is sufficient to show that

$$
\limsup _{k \rightarrow \infty}\left(\prod_{j=1}^{k} \alpha_{e_{j}}\right)^{\frac{1}{k}}<1
$$

Since $\Gamma$ is finite, there are only finitely many paths and cycles and we can define

$$
a:=\max \left\{\alpha(C)^{\frac{1}{|C|}}: C \text { cycle }\right\} \quad \text { and } \quad b:=\max \left\{\alpha(P)^{\frac{1}{|P|}}: P \text { path }\right\} .
$$

Now fix some $k \in \mathbb{N}$ and consider the truncated walk

$$
W^{(k)}=v_{1} \stackrel{e_{1}}{\rightarrow} v_{2} \stackrel{e_{2}}{\longrightarrow} \cdots \stackrel{e_{k}}{\rightarrow} v_{k+1}
$$

We may decompose $W^{(k)}$ into a path from $v_{1}$ to $v_{k+1}$ and finitely many elementary cycles as depicted in Figure 4. If the path has $t \leq k$ edges, the total number of edges in the cycles is $k-t$ and we obtain

$$
\alpha_{W^{(k)}}=\prod_{j=1}^{k} \alpha_{e_{j}} \leq a^{k-t} b^{t}=\left(\frac{b}{a}\right)^{t} a^{k} .
$$

Let $\ell \geq 0$ be the maximal length of a path in $\Gamma$ and set $c=\max (b / a, 1)$ to obtain

$$
\left(\prod_{j=1}^{k} \alpha_{e_{j}}\right)^{\frac{1}{k}} \leq\left(c^{\ell} a^{k}\right)^{\frac{1}{k}}=c^{\frac{\ell}{k}} a \stackrel{k \rightarrow \infty}{\longrightarrow} a .
$$

Since all cycles in $\Gamma$ are gainy by assumption, we have $a<1$, finishing the proof.

Using Proposition 3.2, we can define the desired linear forms. 
Definition 3.3. For $W \in \mathcal{W}$ define an affine-linear form $\Sigma(W): \mathbb{R}^{V} \rightarrow \mathbb{R}$ as follows. If $W$ is a finite walk as in (7), set

$$
\Sigma(W)(x):=\sum_{k=1}^{r}\left(\prod_{j=1}^{k-1} \alpha_{e_{j}}\right)\left(x_{v_{k}}+c_{e_{k}}\right)+\left(\prod_{j=1}^{r} \alpha_{e_{j}}\right) x_{v_{r+1}}
$$

If $W$ is an infinite walk as in (8), set

$$
\Sigma(W)(x):=\sum_{k=1}^{\infty}\left(\prod_{j=1}^{k-1} \alpha_{e_{j}}\right)\left(x_{v_{k}}+c_{e_{k}}\right)
$$

By construction, the defined linear forms satisfy the recursion (9). Indeed (9) together with $\Sigma(a)(x):=$ $\lambda_{a}$ uniquely determines the linear forms $\Sigma(W)$ given the convergence in Proposition 3.2.

Proposition 3.4. For any $x \in \mathbb{R}^{V}$ we have $\sup _{W \in \mathcal{W}} \Sigma(W)(x)<\infty$.

Proof. Let the constants $M, a, b, c, \ell$ be given as in the proof of Proposition 3.2. For finite walks $W \in \mathcal{W}$ as in (7), we have

$$
\Sigma(W)(x)=\sum_{k=1}^{r}\left(\prod_{j=1}^{k-1} \alpha_{e_{j}}\right)\left(x_{v_{k}}+c_{e_{k}}\right)+\left(\prod_{j=1}^{r} \alpha_{e_{j}}\right) x_{v_{r+1}} \leq M \sum_{k=1}^{r+1} c^{\ell} a^{k-1} \leq \frac{M c^{\ell}}{1-a} .
$$

Likewise, for infinite walks as in (8), we have

$$
\Sigma(W)(x)=\sum_{k=1}^{\infty}\left(\prod_{j=1}^{k-1} \alpha_{e_{j}}\right)\left(x_{v_{k}}+c_{e_{k}}\right) \leq M \sum_{k=1}^{\infty} c^{\ell} a^{k-1}=\frac{M c^{\ell}}{1-a}
$$

3.1. The inverse transfer map. We are now ready to construct an inverse to the transfer map $\phi_{\Gamma}$. For $v \in V$ denote by $\mathcal{W}_{v}$ the set of all walks $\gamma \in \mathcal{W}$ starting in $v$.

Theorem 3.5. Let $\Gamma=(V \uplus A, E, \alpha, c, \lambda)$ be a gainy marked network with all sinks marked. The transfer map $\phi_{\Gamma}: \mathbb{R}^{V} \rightarrow \mathbb{R}^{V}$ is a piecewise-linear bijection with inverse $\psi_{\Gamma}: \mathbb{R}^{V} \rightarrow \mathbb{R}^{V}$ given by

$$
\psi_{\Gamma}(y)_{v}:=\sup _{W \in \mathcal{W}_{v}} \Sigma(W)(y)
$$

Since part of the proof of Theorem 3.5 will be relevant when we give a description of $\phi_{\Gamma}(\mathcal{O}(\Gamma))$ below, we provide the following lemma first.

Lemma 3.6. For any $x \in \mathbb{R}^{V}$ and $v \in V \uplus A$ we have

$$
\sup _{W \in \mathcal{W}_{v}} \Sigma(W)\left(\phi_{\Gamma}(x)\right) \leq x_{v}
$$


Proof. Let $y=\phi_{\Gamma}(x)$ for $x \in \mathbb{R}^{V}$. For a finite walk $W \in \mathcal{W}$ as in (7) starting in $v_{1}=v$, we have

$$
\begin{aligned}
\Sigma(W)(y) & =\sum_{k=1}^{r}\left(\prod_{j=1}^{k-1} \alpha_{e_{j}}\right)\left(y_{v_{k}}+c_{e_{k}}\right)+\left(\prod_{j=1}^{r} \alpha_{e_{j}}\right) y_{v_{r+1}} \\
& =\sum_{k=1}^{r}\left(\prod_{j=1}^{k-1} \alpha_{e_{j}}\right)\left(x_{v_{k}}-\max _{v_{k} \rightarrow w}\left(\alpha_{e} x_{w}+c_{e}\right)+c_{e_{k}}\right)+\left(\prod_{j=1}^{r} \alpha_{e_{j}}\right) x_{v_{r+1}} \\
& \leq \sum_{k=1}^{r}\left(\prod_{j=1}^{k-1} \alpha_{e_{j}}\right)\left(x_{v_{k}}-\alpha_{e_{k}} x_{v_{k+1}}\right)+\left(\prod_{j=1}^{r} \alpha_{e_{j}}\right) x_{v_{r+1}} \\
& =x_{v_{1}}=x_{v} .
\end{aligned}
$$

For an infinite walk as in (8) starting in $v_{1}=v$, we have

$$
\begin{aligned}
\Sigma(W)(y) & =\sum_{k=1}^{\infty}\left(\prod_{j=1}^{k-1} \alpha_{e_{j}}\right)\left(y_{v_{k}}+c_{e_{k}}\right) \\
& \leq \sum_{k=1}^{\infty}\left(\prod_{j=1}^{k-1} \alpha_{e_{j}}\right)\left(x_{v_{k}}-\alpha_{e_{k}} x_{v_{k+1}}\right)=x_{v_{1}}=x_{v} .
\end{aligned}
$$

Proof of Theorem 3.5. For $v \in V$, all walks $W \in \mathcal{W}_{v}$ are of the form $v \stackrel{e}{\rightarrow} W^{\prime}$ for an edge $v \stackrel{e}{\rightarrow} w$ and $W^{\prime} \in \mathcal{W}_{w}$. Hence, by the recursive property (9) we have

$$
\Sigma(W)(y)=\alpha_{e} \Sigma\left(W^{\prime}\right)(y)+\left(y_{v}+c_{e}\right) .
$$

We conclude that $\psi$ satisfies the recursion

$$
\psi_{\Gamma}(y)_{v}=y_{v}+\max _{v \rightarrow w}\left(\alpha_{e} \psi_{\Gamma}(y)_{w}+c_{e}\right) \quad \text { for all } v \in V
$$

Comparing this to the definition of $\phi_{\Gamma}$, we see that $\phi_{\Gamma} \circ \psi_{\Gamma}$ is the identity on $\mathbb{R}^{V}$.

Regarding the composition $\psi_{\Gamma} \circ \phi_{\Gamma}$, first note that $\psi_{\Gamma}\left(\phi_{\Gamma}(x)\right)_{v} \leq x_{v}$ for all $v \in V$ by Lemma 3.6. Hence, to show that $\psi_{\Gamma}\left(\phi_{\Gamma}(x)\right)_{v}=x_{v}$ for $v \in V$, it is enough to construct a walk $W \in \mathcal{W}_{v}$ such that $\Sigma(W)\left(\phi_{\Gamma}(x)\right) \geq x_{v}$. Let $v_{1}=v$ and successively pick an edge $v_{k} \stackrel{e_{k}}{\rightarrow} v_{k+1}$ such that

$$
\alpha_{e_{k}} x_{v_{k+1}}+c_{e_{k}}=\max _{v_{k} \rightarrow w}\left(\alpha_{e} x_{w}+c_{e}\right)
$$

until either $v_{k+1}$ is marked or $v_{k+1}$ already appeared in $\left\{v_{1}, \ldots, v_{k}\right\}$.

In the first case we constructed a finite walk $W \in \mathcal{W}_{v}$ as in (7) satisfying

$$
\begin{aligned}
\Sigma(W)\left(\phi_{\Gamma}(x)\right) & =\sum_{k=1}^{r}\left(\prod_{j=1}^{k-1} \alpha_{e_{j}}\right)\left(\phi_{\Gamma}(x)_{v_{k}}+c_{e_{k}}\right)+\left(\prod_{j=1}^{r} \alpha_{e_{j}}\right) \phi_{\Gamma}(x)_{v_{r+1}} \\
& =\sum_{k=1}^{r}\left(\prod_{j=1}^{k-1} \alpha_{e_{j}}\right)\left(x_{v_{k}}-\alpha_{e_{k}} x_{v_{k+1}}\right)+\left(\prod_{j=1}^{r} \alpha_{e_{j}}\right) x_{v_{r+1}}=x_{v_{1}}=x_{v} .
\end{aligned}
$$

In the second case, we ended at an unmarked element $v_{r+1}=v_{s}$ for $s \leq r$. This yields an infinite walk $W \in \mathcal{W}_{v}$ of the form

$$
v_{1} \stackrel{e_{1}}{\longrightarrow} \cdots \stackrel{e_{s-1}}{\longrightarrow} v_{s} \stackrel{e_{s}}{\longrightarrow} \cdots \stackrel{v_{r-1}}{\longrightarrow} e_{r} \stackrel{e_{r}}{\longrightarrow} v_{s} \stackrel{e_{s}}{\longrightarrow} \cdots \stackrel{v_{r-1}}{\longrightarrow} e_{r} \stackrel{e_{r}}{\longrightarrow} v_{s} \stackrel{e_{s}}{\longrightarrow} \cdots
$$




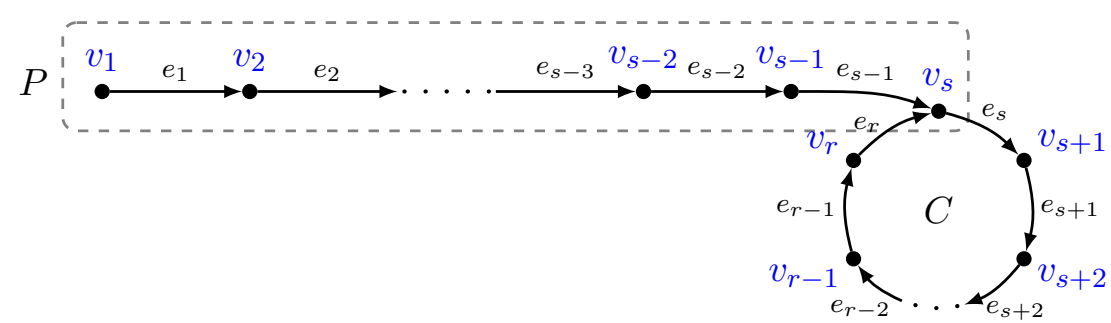

Figure 5. A monocycle. Note that the visible nodes are pairwise distinct.

That is, $W$ walks from $v_{1}$ to $v_{s}$ and then infinitely often runs through the cycle

$$
v_{s} \stackrel{e_{s}}{\longrightarrow} v_{s+1} \stackrel{e_{s+1}}{\longrightarrow} \cdots \stackrel{v_{r-1}}{\longrightarrow} e_{r} \stackrel{e_{r}}{\longrightarrow} v_{s} .
$$

Treating indices $k>r$ accordingly, we obtain

$$
\begin{aligned}
\Sigma(W)\left(\phi_{\Gamma}(x)\right) & =\sum_{k=1}^{\infty}\left(\prod_{j=1}^{k-1} \alpha_{e_{j}}\right)\left(\phi_{\Gamma}(x)_{v_{k}}+c_{e_{k}}\right) \\
& =\sum_{k=1}^{\infty}\left(\prod_{j=1}^{k-1} \alpha_{e_{j}}\right)\left(x_{v_{k}}-\alpha_{e_{k}} x_{v_{k+1}}\right)=x_{v_{1}}=x_{v} .
\end{aligned}
$$

In both cases $\Sigma(W)\left(\phi_{\Gamma}(x)\right)=x_{v}$ and we obtain $\psi_{\Gamma}\left(\phi_{\Gamma}(x)\right)_{v}=x_{v}$ as desired. We conclude that $\phi_{\Gamma}$ and $\psi_{\Gamma}$ are mutually inverse piecewise-linear self-maps of $\mathbb{R}^{V}$.

Inspecting the proof of Theorem 3.5, we see that only a finite subset of $\mathcal{W}$ is necessary to define $\psi_{\Gamma}$. Namely, the paths with only the last node marked and the infinite walks that keep repeating a cycle after a finite number of steps as in (10). We will refer to walks of the latter kind as monocycles and denote them by $W=P * C$, where $P$ is the path and $C$ the cycle. Note that only the end node of $P$ is shared with $C$. A visual representation of a monocycle can be found in Figure 5.

Definition 3.7. Let $\widehat{\mathcal{W}} \subseteq \mathcal{W}$ be the subset of walks $W \in \mathcal{W}$ such that $W$ is either a path or a monocycle as in (10) with pairwise distinct $v_{1}, \ldots, v_{r}$. For $v \in V$, Denote by $\widehat{\mathcal{W}}_{v}=\widehat{\mathcal{W}} \cap \mathcal{W}_{v}$ the set of walks in $\widehat{\mathcal{W}}$ starting in $v$.

Corollary 3.8. The inverse transfer map $\psi_{\Gamma}: \mathbb{R}^{V} \rightarrow \mathbb{R}^{V}$ is given by

$$
\psi_{\Gamma}(y)_{v}=\max _{W \in \widehat{\mathcal{W}}_{v}} \Sigma(W)(y) .
$$

Since some of the $W \in \widehat{\mathcal{W}}_{v}$ appearing in this description of the inverse transfer map might be monocycles, we want to give a finite expression for the linear form $\Sigma(W)$.

Proposition 3.9. Let $W=P * C \in \widehat{\mathcal{W}}$ be a monocycle with

$$
\begin{aligned}
& P=v_{1} \stackrel{e_{1}}{\longrightarrow} \cdots \stackrel{e_{s-1}}{\longrightarrow} v_{s}, \\
& C=v_{s} \stackrel{e_{s}}{\longrightarrow} \cdots \stackrel{e_{r-1}}{\longrightarrow} v_{r} \stackrel{e_{r}}{\longrightarrow} v_{s} .
\end{aligned}
$$

Then for all $x \in \mathbb{R}^{V}$ we have

$$
\Sigma(W)(x)=\sum_{k=1}^{s-1}\left(\prod_{j=1}^{k-1} \alpha_{e_{j}}\right)\left(x_{v_{k}}+c_{e_{k}}\right)+\frac{\alpha(P)}{1-\alpha(C)} \sum_{k=s}^{r}\left(\prod_{j=s}^{k-1} \alpha_{e_{j}}\right)\left(x_{v_{k}}+c_{e_{k}}\right) .
$$


Proof. The infinite series in Definition 3.3 yields that $\Sigma(W)(x)$ is equal to

$$
\sum_{k=1}^{s-1}\left(\prod_{j=1}^{k-1} \alpha_{e_{j}}\right)\left(x_{v_{k}}+c_{e_{k}}\right)+\alpha(P) \sum_{l=0}^{\infty}\left[\alpha(C)^{l} \sum_{k=s}^{r}\left(\prod_{j=s}^{k-1} \alpha_{e_{j}}\right)\left(x_{v_{k}}+c_{e_{k}}\right)\right] \text {. }
$$

Since all cycles in $\Gamma$ are gainy, we have $\alpha(C)<1$ and the geometric series $\sum_{l=0}^{\infty} \alpha(C)^{l}$ converges to $(1-\alpha(C))^{-1}$.

Let $x \in \mathbb{R}^{V}$. Now for every $v \in V$, select an edge $v \rightarrow w$ which attains the maximum in the definition of $\phi_{\Gamma}(x)_{v}$ in (5). It becomes clear from our discussion that this yields a marked subnetwork $\Gamma_{x}$ composed of paths and monocycles. More precisely, deleting the cycles, leaves a rooted forest, that is, an acyclic digraph in which every nodes has one edge pointing out. This network realizes $\phi_{\Gamma}$ as an affine-linear function at $x$. The matrix $B=B\left(\Gamma_{x}\right) \in \mathbb{R}^{V \times V}$ with $B_{w w}=1$ and

$$
B_{w v}=-\alpha_{e} \text { if } w \stackrel{e}{\rightarrow} v
$$

and 0 otherwise determines the linear part of $\phi_{\Gamma}$ at $x$. The determinant of $B$ is $\prod_{C}(1-\alpha(C))$ where $C$ ranges over the cycles in $\Gamma_{x}$.

Corollary 3.10. Let $\Gamma=(V \uplus A, E, \alpha, c, \lambda)$ be a gainy marked network with all sinks marked. If $\alpha(C)=2$ for all cycles $C$, then $\phi_{\Gamma}$ is volume preserving. If the weights $\alpha$ and $c$ are integral, then $\phi_{\Gamma}$ is lattice-preserving if and only if for every cycle there is a unique edge $e^{\prime}$ with weight $\alpha_{e^{\prime}}=2$ and all other edges have weight $\alpha_{e}=1$.

\section{Anti-Blocking images}

In the previous section, we showed that distributive polyhedra given by gainy marked networks with at least all sinks marked admit a piecewise-linear bijective transfer map $\phi_{\Gamma}: \mathbb{R}^{V} \rightarrow \mathbb{R}^{V}$ analogous to the transfer map for (marked) order polytopes. In this section we keep the same premise and focus on the image $\phi_{\Gamma}(\mathcal{O}(\Gamma))$. We show that it is an anti-blocking polyhedron with describing inequalities given by the walks in $\widehat{\mathcal{W}}$, similar to the chain polytope being described by inequalities given by chains in the poset.

Definition 4.1. Let $\Gamma=(V \uplus A, E, \alpha, c, \lambda)$ be a gainy marked network with at least all sinks marked. The polyhedron $\mathcal{C}(\Gamma)$ is the set of all $y \in \mathbb{R}^{V}$ with $y \geq 0$ and

$$
\alpha_{e} \Sigma(W)(y)+c_{e} \leq \lambda_{a}
$$

for each walk $a \stackrel{e}{\rightarrow} W$ with $a \in A$ and $W \in \widehat{\mathcal{W}}$.

Theorem 4.2. Let $\Gamma=(V \uplus A, E, \alpha, c, \lambda)$ be a gainy marked network with at least all sinks marked. The transfer map $\phi_{\Gamma}$ restricts to a piecewise-linear homeomorphism $\mathcal{O}(\Gamma) \rightarrow \mathcal{C}(\Gamma)$.

Proof. To show that $\phi_{\Gamma}(\mathcal{O}(\Gamma)) \subseteq \mathcal{C}(\Gamma)$, let $y=\phi_{\Gamma}(x)$ for $x \in \mathcal{O}(\Gamma)$. By definition of $\phi_{\Gamma}$ we have $y_{v} \geq 0$ for $v \in V$. Now let $a \stackrel{e}{\rightarrow} W$ be a walk with $a \in A$ and $W \in \widehat{\mathcal{W}}$. It follows from Lemma 3.6 that

$$
\alpha_{e} \Sigma(W)(y)+c_{e} \leq \alpha_{e} x_{v}+c_{e} \leq x_{a}=\lambda_{a} .
$$

Now let $y$ be any point in $\mathcal{C}(\Gamma)$ and let $x=\psi_{\Gamma}(y)$. For any edge $v \stackrel{e}{\rightarrow} w$ we have to show that $\alpha_{e} x_{w}+c_{e} \leq x_{v}$. Let $W \in \widehat{\mathcal{W}}_{w}$ be a walk starting in $w$ constructed as in the proof of Theorem 3.5 such that $\Sigma(W)(y)=x_{w}$. If $v \in V$, we can again appeal to Lemma 3.6 together with $y \geq 0$ to obtain

$$
\alpha_{e} x_{w}+c_{e}=\alpha_{e} \Sigma(W)(y)+c_{e}=\Sigma(v \stackrel{e}{\rightarrow} W)(y)-x_{v} \leq \Sigma(v \stackrel{e}{\rightarrow} W)(y) \leq x_{v} .
$$

Otherwise, if $v \in A$, the walk $v \stackrel{e}{\rightarrow} W$ appears in Definition 4.1, so that

$$
\alpha_{e} x_{w}+c_{e}=\alpha_{e} \Sigma(W)(y)+c_{e} \leq \lambda_{v}=x_{v} .
$$


Fulkerson [14] introduced anti-blocking polyhedra and gave the following characterization.

Proposition 4.3 ([14]). A polyhedron $Q \subseteq \mathbb{R}_{\geq 0}^{d}$ is anti-blocking if and only if there are $a_{1}, \ldots, a_{m} \in$ $\mathbb{R}_{\geq 0}^{d}$ and $b_{1}, \ldots, b_{m} \in \mathbb{R}_{\geq 0}$ such that

$$
Q=\left\{x \in \mathbb{R}_{\geq 0}^{d}: a_{i}^{t} x \leq b_{i} \text { for } i=1, \ldots, m\right\} .
$$

This description allows us to prove the following.

Corollary 4.4. The polyhedron $\mathcal{C}(\Gamma)$ is anti-blocking.

Proof. By definition $\mathcal{C}(\Gamma) \subseteq \mathbb{R}_{\geq 0}^{V}$. Furthermore, the coefficients in an inequality $\alpha_{e} \Sigma(\gamma)(y)+c_{e} \leq \lambda_{a}$ are all non-negative: for finite walks they are just finite products of edge weights $\alpha_{e^{\prime}}$ while for monocycles some of them are multiplied by the positive factor $\alpha(P) /(1-\alpha(C))$ as described in Proposition 3.9.

Example 4.5 (continuation of Example 2.5). Recall the marked network $\Gamma$ with two unmarked nodes depicted in Figure 3 together with the distributive polytope $\mathcal{O}(\Gamma)$ and its anti-blocking image now denoted by $\mathcal{C}(\Gamma)$. We label the three edges between $v$ and $w$ from top to bottom by $e, f, g$.

Since $\Gamma$ does not have marked nodes with incoming edges and all cycles contain only unmarked nodes, the set of monocycles $\widehat{\mathcal{W}}$ is given by the cycles with trivial acyclic beginning:

$$
\begin{array}{ll}
W_{1}=v \stackrel{e}{\rightarrow} w \stackrel{g}{\rightarrow} v \stackrel{e}{\rightarrow} w \stackrel{g}{\rightarrow} \cdots & W_{2}=v \stackrel{f}{\rightarrow} w \stackrel{g}{\rightarrow} v \stackrel{f}{\rightarrow} w \stackrel{g}{\rightarrow} \cdots \\
W_{3}=w \stackrel{g}{\rightarrow} v \stackrel{e}{\rightarrow} w \stackrel{g}{\rightarrow} v \stackrel{e}{\rightarrow} \cdots & W_{4}=w \stackrel{g}{\rightarrow} v \stackrel{f}{\rightarrow} w \stackrel{g}{\rightarrow} v \stackrel{f}{\rightarrow} \cdots
\end{array}
$$

From Proposition 3.9 with trivial acyclic beginning $(s=1)$ we obtain

$$
\begin{array}{ll}
\Sigma\left(W_{1}\right)(y)=\frac{4}{3} x_{v}+\frac{2}{3} x_{w} & \Sigma\left(W_{2}\right)(y)=2 x_{v}+2 x_{w}-2 \\
\Sigma\left(W_{3}\right)(y)=\frac{2}{3} x_{v}+\frac{4}{3} x_{w} & \Sigma\left(W_{4}\right)(y)=x_{v}+2 x_{w}-1
\end{array}
$$

Hence, the inverse transfer map on $\mathbb{R}^{V}$ is given by

$$
\psi_{\Gamma}\left(\begin{array}{c}
y_{v} \\
y_{w}
\end{array}\right)=\left(\begin{array}{c}
\max \left\{\frac{4}{3} x_{v}+\frac{2}{3} x_{w}, 2 x_{v}+2 x_{w}-2\right\} \\
\max \left\{\frac{2}{3} x_{v}+\frac{4}{3} x_{w}, x_{v}+2 x_{w}-1\right\}
\end{array}\right) .
$$

Note that the linearity regions are the two half-spaces given by the hyperplane $\frac{1}{3} x_{v}+\frac{2}{3} x_{w}=1$ containing the dashed line in Figure 3c. For the anti-blocking image $\mathcal{C}(\Gamma)$ the only walks appearing in Definition 4.1 are $2 \rightarrow W_{1}$ and $2 \rightarrow W_{2}$ giving inequalities

$$
\frac{4}{3} x_{v}+\frac{2}{3} x_{w} \leq 2 \text { and } 2 x_{v}+2 x_{w} \leq 4
$$

These correspond to the two non-trivial facets in Figure 3c.

In Example 2.4, where we have a lossy cycle and the transfer map is not injective, the image was still an anti-blocking polytope. However, this is not true in general: in the following example we have a lossy cycle, an injective transfer map nevertheless, but the image $\phi_{\Gamma}(\mathcal{O}(\Gamma))$ is not anti-blocking.

Example 4.6. Let $\Gamma$ be the marked network shown in Figure 6a. The distributive polyhedron $\mathcal{O}(\Gamma)$ is the unbounded polyhedron in Figure $6 \mathrm{~b}$ given by the inequalities $2 x_{v}-4 \leq x_{w}, 2 x_{w}-4 \leq x_{v}$, $x_{v} \leq 3$ and $x_{w} \leq 3$. The transfer map is given by

$$
\phi_{\Gamma}\left(\begin{array}{l}
x_{v} \\
x_{w}
\end{array}\right)=\left(\begin{array}{l}
x_{v}-2 x_{w}+4 \\
x_{w}-2 x_{v}+4
\end{array}\right) .
$$

Thus, the image $\phi_{\Gamma}(\mathcal{O}(\Gamma))$ is the polyhedron given by inequalities $0 \leq y_{v}, 0 \leq y_{w}, y_{v}+2 y_{w} \geq 3$ and $2 y_{v}+y_{w} \geq 3$. It is depicted in Figure $6 \mathrm{c}$ and is not an anti-blocking polyhedron. In fact it is what is called a blocking polyhedron in [14]: it is given given by inequalities $x_{i} \geq 0$ for all coordinates together with inequalities of the form $a^{t} x \geq 1$ with $a \in \mathbb{R}_{\geq 0}^{n}$. 


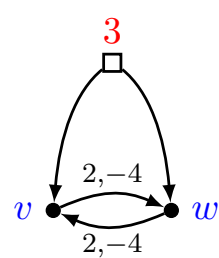

(a) a network $\Gamma$

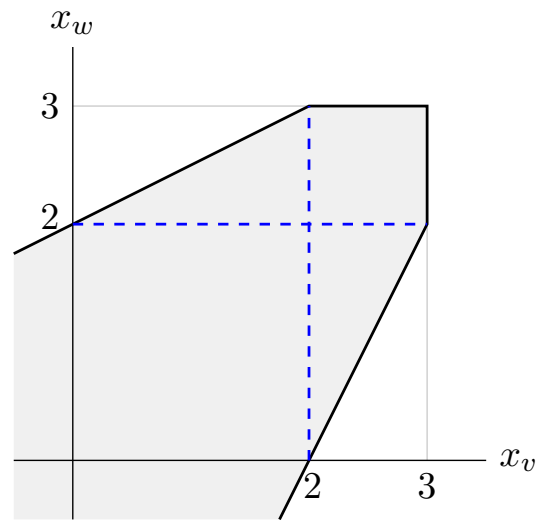

(b) the polyhedron $\mathcal{O}(\Gamma)$

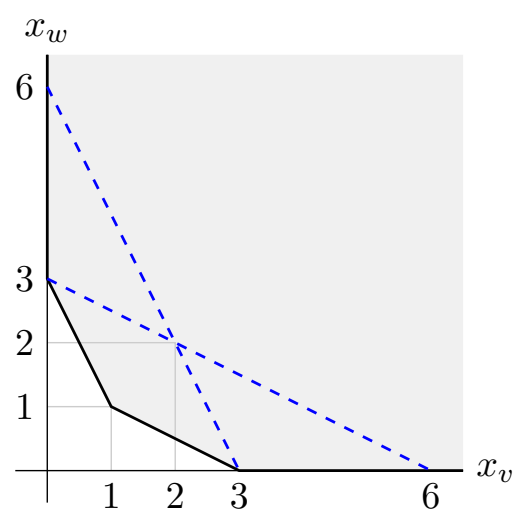

(c) the image $\phi_{\Gamma}(\mathcal{O}(\Gamma))$

Figure 6. The marked network $\Gamma$ of Example 4.6 with the associated distributive polyhedron and its non-anti-blocking image under the transfer map.

4.1. Duality. Let $Q \subseteq \mathbb{R}^{n}$ be a distributive polyhedron. It follows from the definition that $-Q$ is distributive as well. If $Q=\mathcal{O}(\Gamma)$, then $-Q=\mathcal{O}\left(\Gamma^{\mathrm{op}}\right)$, where $\Gamma^{\mathrm{op}}=\left(V \uplus A, E^{\prime}, \alpha^{\prime}, c^{\prime}, \lambda^{\prime}\right)$ is the opposite network with edges $w \stackrel{e^{\prime}}{\rightarrow} v$ for each edge $v \stackrel{e}{\rightarrow} w$ in $\Gamma$, weights $\alpha_{e^{\prime}}=\frac{1}{\alpha_{e}}, c_{e^{\prime}}=\frac{c_{e}}{\alpha_{e}}$, and $\lambda^{\prime}=-\lambda$. If $(P, \preceq)$ is a poset, then $-\mathcal{O}(P)$ is, up to a translation, the order polytope $\mathcal{O}\left(P^{\mathrm{op}}\right)$, where $P^{\mathrm{op}}$ is the opposite poset.

If $\Gamma$ is a network with all sources marked, then $\phi_{\Gamma}^{\mathrm{op}}: \mathbb{R}^{V} \rightarrow \mathbb{R}^{V}$ given by $\phi_{\Gamma}^{\mathrm{op}}(x):=\phi_{\Gamma \text { op }}(-x)$ is a piecewise-linear map. More precisely, it is given by

$$
\phi_{\Gamma}^{\mathrm{op}}(x)_{v}=-x_{v}+\min _{w e_{v}}\left(\frac{1}{\alpha_{e}} x_{w}-\frac{c_{e}}{\alpha_{e}}\right) .
$$

If $\Gamma$ has only lossy cycles, then $\phi_{\Gamma}^{\text {op }}$ is bijective and restricts to a homeomorphism $\mathcal{O}(\Gamma) \rightarrow \mathcal{C}\left(\Gamma^{\mathrm{op}}\right)$.

When $\Gamma$ is acyclic and both all sinks and all sources are marked, we can compare the anti-blocking polyhedra $\mathcal{C}(\Gamma)$ and $\mathcal{C}\left(\Gamma^{\mathrm{op}}\right)$. If $\Gamma$ is the Hasse diagram of a poset, we have $\mathcal{C}(\Gamma)=\mathcal{C}\left(\Gamma^{\mathrm{op}}\right)$ as a consequence of the opposite poset having the same comparability graph. By comparing the defining inequalities of the two polyhedra in the general case, we can see that this observation still holds for arbitrary acyclic marked networks with all sinks and sources marked.

\section{Applichtions and Questions}

5.1. Cayley polytopes. Recall that the Cayley polytope $C_{n}$ is the distributive polytope $\mathcal{O}(\Gamma)$ associated to the marked network in Figure 1. The geometric bijection in [18] is a linear transformation $\phi^{-1}: C_{n} \rightarrow \mathbf{Y}_{n}$, where $\mathbf{Y}_{n}$ is an anti-blocking polytope defined in the introduction. This map is exactly the transfer map $\psi_{\Gamma}^{\mathrm{op}}: \mathcal{C}(\Gamma) \rightarrow \mathcal{O}(\Gamma)$.

5.2. Lecture hall order cones and polytopes. The $s$-lecture hall cones and polytopes of BousquetMélou and Eriksson [5, 6] and Stanley's P-partitions [23, Sect. 3.15] were elegantly combined in [7] to lecture hall order cones/polytopes. Here we briefly sketch a generalization to a marked version. Let $(P, \preceq, \lambda)$ be a marked poset with $\lambda \in \mathbb{R}^{A}$ for $A \subseteq P$. For any $s \in \mathbb{R}_{>0}^{P}$, define the marked lecture hall order polyhedron $\mathcal{O}(P, \lambda, s)$ as the set of points $x \in \mathbb{R}^{P \backslash A}$

$$
\frac{x_{p}}{s_{p}} \leq \frac{x_{q}}{s_{q}} \text { for } p \prec q,
$$


where we set $x_{a}=s_{a} \lambda_{a}$ for $a \in A$. If $s \equiv 1$, then $\mathcal{O}(P, \lambda, s)$ is the marked order polyhedron $\mathcal{O}(P, \lambda)$. When $P$ is the linear poset $\hat{0} \prec p_{1} \prec \cdots \prec p_{n}$ and $\lambda_{\hat{0}}=0$, we recover the $s$-lecture hall cones and adding a maximal element $\hat{1}$ with marking $\lambda_{\hat{1}}=1$ we get the $s$-lecture hall polytopes.

Note that $\mathcal{O}(P, \lambda, s)=\mathcal{O}(\Gamma)$ for the marked network given by the Hasse diagram of $P$ with edge weights $c \equiv 0$ and $\alpha_{e}=\frac{s_{q}}{s_{p}}$ for an edge $e$ given by a covering relation $p \prec q$. We may also express $\mathcal{O}(P, \lambda, s)$ as a linear transformation $T_{s}(\mathcal{O}(P, \lambda))$ of the usual marked order polyhedron, where $T_{s}(x)_{p}=s_{p} x_{p}$. This transformation is compatible with the transfer maps associated to $\mathcal{O}(P, \lambda)$ and $\mathcal{O}(P, \lambda, s)=\mathcal{O}(\Gamma)$ in the sense that $T_{s} \circ \phi_{(P, \lambda)}=\phi_{\Gamma} \circ T_{s}$. If $\lambda$ and $s$ are integral, then the marked lecture hall order polytopes are lattice polytopes. Furthermore, if $s$ satisfies $s_{p} \mid s_{q}$ for $p \prec q$, then the transfer map is lattice preserving.

5.3. Coordinates in polytopes. The geometric reformulation (6) admits the following generalization: Given a polyhedron $Q \subseteq \mathbb{R}^{d}$ and vectors $U=\left(u_{1}, \ldots, u_{m}\right) \in \mathbb{R}^{d \times m}$. Define $\phi_{Q, U}: Q \rightarrow \mathbb{R}^{m}$ by

$$
\phi_{Q, U}(x)_{i}:=\max \left(\mu \geq 0: x-\mu v_{i} \in Q\right) .
$$

Question 5.1. For which $(Q, U)$ is $\phi_{Q, U}$ injective? When is the image convex?

If $\phi_{Q, U}$ is injective and convex, then its image gives a representation of $Q$ up to translation, akin to its slack representation; see [16, Sect. 3.2]. Our results show that $\phi_{\Gamma}$ yields a class of examples for gainy networks. However, Example 4.6 shows that even for some distributive polyhedra associated to marked networks with non-gainy cycles the transfer map can still be injective.

5.4. Continuous families. In [9], Fang and Fourier generalized the marked poset polytopes $\mathcal{O}(P, \lambda)$ and $\mathcal{C}(P, \lambda)$ to a discrete family of marked chain-order polytopes $\mathcal{O}_{C, O}(P, \lambda)$. It is parametrized by partitions $P \backslash A=C \uplus O$ such that $C=\varnothing$ yields the order polytope, which is distributive, and $O=\varnothing$ yields the chain polytope, which is anti-blocking. When both $C$ and $O$ are non-empty, $\mathcal{O}_{C, O}(P, \lambda)$ is neither distributive nor anti-blocking in general.

Definition 5.2. Let $D$ and $A$ be finite sets. A polyhedron $Q \subseteq \mathbb{R}^{D} \times \mathbb{R}_{\geq 0}^{A}$ is called mixed distributive anti-blocking if it satisfies the following properties:

i) given $(x, z) \in Q$ and $(y, z) \in Q$, we have $(x \wedge y, z) \in Q$ and $(x \vee y, z) \in Q$,

ii) when $(x, z) \in Q$ and $0 \leq y \leq z$, then $(x, y) \in Q$.

When $A=\varnothing$ or $D=\varnothing$, this recovers the notions of distributive and anti-blocking polyhedra, respectively. The marked chain-order polytopes are then mixed distributive anti-blocking with respect to the decomposition $\mathbb{R}^{P \backslash A}=\mathbb{R}^{O} \times \mathbb{R}^{C}$.

This discrete family of marked chain-order polytopes has been embedded into a continuous family of polytopes $\mathcal{O}_{t}(P, \lambda)$ parametrized by $t \in[0,1]^{P \backslash A}$ in [10]. The marked chain-order polytopes are obtained for characteristic functions $t=\chi_{C}$. These polytopes are all obtained as images of the marked order polytope $\mathcal{O}(P, \lambda)$ under parametrized transfer maps

$$
\phi_{t}(x)_{p}:=x_{p}-t_{p} \cdot \max _{q \prec p} x_{q} .
$$

Hence, it is natural to ask whether we can obtain an analogous continuous family of polyhedra associated to marked networks.

Question 5.3. Does introducing a parameter $t \in[0,1]^{V}$ in the transfer map of distributive polyhedra associated to gainy marked networks with marked sinks yield a continuous family of polyhedra such that 
i) the combinatorial type of the images is constant along relative interiors of the parametrizing hypercube and

ii) the polyhedra at the vertices of the hypercube are mixed distributive anti-blocking?

5.5. Domains of linearity, faces, Minkowski summands. At the end of Section 3.1, we gave an idea of the domains of linearity of $\phi_{\Gamma}$. They are related to rooted forests with cycles attached to some leafs. Stanley [22] considered a refined subdivision of $\mathcal{O}(P)$ that had the property of being unimodular. For marked order polytopes a corresponding subdivision was described in [15] in terms of products of dilated unimodular simplices. In the general case with arbitrary weights it is not clear if such fine subdivisions exist.

Question 5.4. Do distributive polyhedra admit a natural subdivision into products of simplices on which the transfer map is linear?

The face structure of marked order polyhedra can be described by so-called face partitions $[22,15,21]$. The question of describing the vertices of $\mathcal{O}(\Gamma)$ was also raised in [12].

Question 5.5. Give a combinatorial description of the faces of $\mathcal{O}(\Gamma)$ in terms of the underlying network.

In $[13,21]$, a marked poset is called regular if the inequalities derived from the cover relations are irredundant (or facet-defining).

Question 5.6. When is a marked network regular?

Finally, polyhedra may be decomposed into Minkowski summands. For marked order polyhedra this was done in [15, 21], for marked chain-order polyhedra in $[9,11]$.

Question 5.7. Is there a Minkowski sum decomposition of distributive polyhedra similar to the one for marked order polyhedra and marked chain-order polyhedra?

\section{REFERENCES}

[1] F. Ardila, T. Bliem, And D. Salazar, Gelfand-Tsetlin polytopes and Feigin-Fourier-Littelmann-Vinberg polytopes as marked poset polytopes, J. Combin. Theory Ser. A, 118 (2011), pp. 2454-2462. 1, 3

[2] M. Beck, B. Braun, AND N. LE, Mahonian partition identities via polyhedral geometry, in From Fourier analysis and number theory to Radon transforms and geometry, vol. 28 of Dev. Math., Springer, New York, 2013, pp. 41-54. 2

[3] M. Beck and R. Sanyal, Combinatorial Reciprocity Theorems, vol. 195 of Graduate Studies in Mathematics, American Mathematical Society, Providence, RI, 2018. 1

[4] B. Bollobás And G. R. Brightwell, Convex bodies, graphs and partial orders, Proc. London Math. Soc. (3), 80 (2000), pp. 415-450. 2

[5] M. Bousquet-Mélou And K. Eriksson, Lecture hall partitions, The Ramanujan Journal, 1 (1997), pp. $101-111$. 2,12

[6] - Lecture hall partitions II, The Ramanujan Journal, 1 (1997), pp. 165-185. 2, 12

[7] P. BRÄNDÉn AND M. LEANDER, Lecture hall p-partitions. https://arxiv.org/abs/1609.02790, September 2016. 2,12

[8] T. Chappell, T. Friedl, and R. Sanyal, Two double poset polytopes, SiAM J. Discrete Math., 31 (2017), pp. 2378-2413. 1

[9] X. FAng AND G. FourIER, Marked chain-order polytopes, European J. Combin., 58 (2016), pp. 267-282. 1, 13, 14

[10] X. Fang, G. Fourier, J.-P. Litza, and C. Pegel, A continuous family of marked poset polytopes. https: //arxiv.org/abs/1712.01037, December 2017. 1, 13

[11] X. FAng, G. Fourier, And C. PEgel, The Minkowski property and reflexivity of marked poset polytopes. https: //arxiv.org/abs/1807.03970, July 2018. 14

[12] S. Felsner And K. Knauer, Distributive lattices, polyhedra, and generalized flows, European Journal of Combinatorics, 32 (2011), pp. 45-59. 2, 3, 5, 14 
[13] G. Fourier, Marked poset polytopes: Minkowski sums, indecomposables, and unimodular equivalence, Journal of Pure and Applied Algebra, 220 (2016), pp. 606-620. 14

[14] D. R. Fulkerson, Blocking and anti-blocking pairs of polyhedra, Mathematical Programming, 1 (1971), pp. 168194. 2,11

[15] K. Jochemko and R. Sanyal, Arithmetic of marked order polytopes, monotone triangle reciprocity, and partial colorings, SIAM J. Discrete Math., 28 (2014), pp. 1540-1558. 1, 14

[16] V. KAIBEL, Extended formulations in combinatorial optimization. Optima 85, 2011. 14 pages. 13

[17] M. Konvalinka And I. PaK, Triangulations of Cayley and Tutte polytopes, Adv. Math., 245 (2013), pp. 1-33. 2

[18] - Cayley compositions, partitions, polytopes, and geometric bijections, J. Combin. Theory Ser. A, 123 (2014), pp. 86-91. 2, 12

[19] T. Lam And A. Postnikov, Alcoved polytopes. I, Discrete Comput. Geom., 38 (2007), pp. 453-478. 2

[20] C. PEGEL, The polyhedral geometry of partially ordered sets, PhD thesis, Universität Bremen, Dissertation, 2017, Bremen, 2017. https://nbn-resolving.de/urn:nbn:de:gbv:46-00106282-13. 2

[21] C. Pegel, The face structure and geometry of marked order polyhedra, Order, 35 (2018), pp. 467-488. 1, 3, 14

[22] R. P. Stanley, Two poset polytopes, Discrete Comput. Geom., 1 (1986), pp. 9-23. 1, 3, 14

[23] - Enumerative combinatorics. Volume 1, vol. 49 of Cambridge Studies in Advanced Mathematics, Cambridge University Press, Cambridge, second ed., 2012. 12

[24] R. P. Stanley and J. Pitman, A polytope related to empirical distributions, plane trees, parking functions, and the associahedron, Discrete Comput. Geom., 27 (2002), pp. 603-634. 2

Institut für Algebra, Zahlentheorie und Diskrete Mathematik, Leibniz Universität Hannover, GerMANY

E-mail address: pegel@math.uni-hannover.de

Institut für Mathematik, Goethe-Universität Frankfurt, Germany

E-mail address: sanyal@math.uni-frankfurt.de 